\title{
Detection of minimal residual disease identifies differences in treatment response between T-ALL and precursor B-ALL
}

Marja J. Willemse, Taku Seriu, Klaudia Hettinger, Elisabetta d'Aniello, Wim C. J. Hop, E. Renate Panzer-Grümayer, Andrea Biondi, Martin Schrappe, Willem A. Kamps, Guiseppe Masera, Helmut Gadner, Hansjoerg Riehm, Claus R. Bartram, and Jacques J. M. van Dongen

\begin{abstract}
We performed sensitive polymerase chain reaction-based minimal residual disease (MRD) analyses on bone marrow samples at 9 follow-up time points in 71 children with T-lineage acute lymphoblastic leukemia (T-ALL) and compared the results with the precursor $B$-lineage ALL (B-ALL) results ( $n=210$ ) of our previous study. At the first 5 follow-up time points, the frequency of MRD-positive patients and the MRD levels were higher in T-ALL than in precursor-B-ALL, reflecting the more frequent occurrence of resistant disease in T-ALL. Subsequently, patients were classified according to their MRD level at time point 1 (TP1), taken at the end of induc-
\end{abstract}

tion treatment (5 weeks), and at TP2 just before the start of consolidation treatment (3 months). Patients were considered at low risk if TP1 and TP2 were MRD negative and at high risk if MRD levels at TP1 and TP2 were $10^{-3}$ or higher; remaining patients were considered at intermediate risk. The relative distribution of patients with T-ALL $(n=43)$ over the MRDbased risk groups differed significantly from that of precursor B-ALL $(n=109)$. Twenty-three percent of patients with TALL and $46 \%$ of patients with precursor B-ALL were classified in the low-risk group $(P=.01)$ and had a 5-year relapsefree survival (RFS) rate of $98 \%$ or greater.
In contrast, $28 \%$ of patients with T-ALL were classified in the MRD-based highrisk group compared to only $11 \%$ of patients with precursor B-ALL $(P=.02)$, and the RFS rates were $0 \%$ and $25 \%$, respectively $(P=.03)$. Not only was the distribution of patients with T-ALL different over the MRD-based risk groups, the prognostic value of MRD levels at TP1 and TP2 was higher in T-ALL (larger RFS gradient), and consistently higher RFS rates were found for MRD-negative T-ALL patients at the first 5 follow-up time points. (Blood. 2002;99:4386-4393)

ㄱ) 2002 by The American Society of Hematology

\section{Introduction}

Childhood acute lymphoblastic leukemia (ALL) has a B-lineage origin in approximately $85 \%$ of patients and a T-lineage origin in the remaining approximately $15 \%$ of patients. ${ }^{1}$ Typical T-lineage ALL (T-ALL) is diagnosed in male adolescents and is frequently characterized by hyperleukocytosis, mediastinal mass, and central nervous system involvement. ${ }^{2,3}$ Children with T-ALL generally have a poorer prognosis than those with precursor B-lineage ALL (B-ALL). ${ }^{1,4}$ In T-ALL, the following characteristics were shown to have a negative effect on treatment outcome: patient age 15 years or older, L2 blast morphology, karyotype other than hyperdiploidy, and $\mathrm{CD}^{+}{ }^{+2,3}$ The most immature T-ALLs (CD1-/ $\mathrm{CD}^{-}$) are also associated with unfavorable outcome, whereas $\mathrm{CD}^{+}{ }^{+} \mathrm{T}-\mathrm{ALL}$ forms a distinct subgroup with an excellent prognosis on intensive high-risk treatment. ${ }^{4-7}$ Early in vivo response has been identified as one of the strongest prognostic factors in childhood ALL. ${ }^{8-12}$ Patients with T-ALL frequently have a poor in vivo steroid response $\mathrm{e}^{9,12,13}$ and are resistant to many drugs, as demonstrated by in vitro assays. ${ }^{14}$ Nevertheless, treatment outcome has significantly improved, and the sustained

From the Departments of Immunology and of Epidemiology and Biostatistics, University Hospital Rotterdam/Erasmus University Rotterdam; Dutch Childhood Leukemia Study Group, The Hague; Department of Pediatrics, University Hospital Groningen, The Netherlands; Institute of Human Genetics, University of Heidelberg; Department of Pediatrics, Medizinische Hochschule Hannover, Germany; Children's Cancer Research Institute, St Anna Kinderspital, Vienna, Austria; and Department of Pediatrics, University of Milano, Ospedale S. Gerardo, Monza, Italy.

Submitted December 27, 2000; accepted February 20, 2002.

Supported by the BIOMED-1 program of the European Commission (grant BMH-CMT 94-1675), Dutch Cancer Society/Koningin Wilhelmina Fonds (grants EUR 94-852 and SNWLK 97-1567), Ank Van Vlissingen Founda- relapse-free survival (RFS) rate is $60 \%$ to $70 \%$ in patients with T-ALL on intensive chemotherapy. ${ }^{15-17}$

Individualization of ALL treatment might further improve outcome and long-term quality of life. This may be achieved through minimal residual disease (MRD) studies that allow the sensitive detection of leukemic cells undetectable by normal cytomorphologic examination, thereby providing accurate information about the in vivo efficacy of cytotoxic treatment. ${ }^{18,19}$ The most broadly applicable MRD technique in ALL is polymerase chain reaction (PCR) analysis of clone-specific immunoglobulin and T-cell receptor (TCR) gene rearrangements, which are easily identified in most pediatric and adult patients with ALL. ${ }^{19-21}$ Based on the sequences of the patient-specific junctional regions, sensitivities of $10^{-4}$ to $10^{-5}$ (one leukemic cell in 10000 to 100000 normal cells) are reached. ${ }^{22}$

Several large-scale studies in childhood ALL have shown that MRD analysis can predict outcome by determining the reduction of the leukemic cell burden during the first months of therapy. ${ }^{23-27}$ Multivariate analysis showed that MRD information is an important

tion (M.J.W., J.J.M.v.D.), Deutsche Krebshilfe (C.R.B.), M. Tettamanti Foundation (A.B., G.M., E.D.), Associazione Italiana per la Ricerca sul Cancro, Minestero Università e Ricerca Scientifica e Technologica, Österreichische Kinderkrebshilfe (E.R.P.-G., K.H.), and FWF P-13575-MED (E.R.P.-G.).

Reprints: J. J. M. van Dongen, Department of Immunology, Erasmus University Rotterdam, Dr Molewaterplein 50, 3015 GE Rotterdam, The Netherlands; e-mail: vandongen@immu.fgg.eur.nl.

The publication costs of this article were defrayed in part by page charge payment. Therefore, and solely to indicate this fact, this article is hereby marked "advertisement" in accordance with 18 U.S.C. section 1734.

(C) 2002 by The American Society of Hematology 
prognostic factor at all follow-up time points taken during and after treatment and that this MRD information is independent of the classical clinical parameters at diagnosis such as age, sex, white blood cell count (WBC), immunophenotype, chromosome aberrations, and prednisone response. ${ }^{25-27}$ For example, prognostically relevant subgroups such as T-ALL or ALL characterized by $\mathrm{t}(9 ; 22)$, $\mathrm{t}(12,21)$, or $\mathrm{t}(4 ; 11)$ are still heterogeneous in treatment response, ${ }^{2,28-31}$ but MRD information during treatment of these leukemia subtypes is more discriminative in predicting treatment outcome. . $6,32,33^{\text {Th }}$

The impact of MRD information in childhood ALL appeared to be superior to classical treatment group classification at diagnosis, as was demonstrated by the study of the International BFM Study Group (I-BFM-SG). In this study, a precise risk group classification was achieved by combining sensitive $\left(10^{-4}\right.$ or less $)$ MRD information of 2 follow-up time points (TP): TP1, at the end of induction treatment, and TP2, just before the start of consolidation treatment. ${ }^{27}$ Three risk groups were identified: a low-risk group consisting of $43 \%$ of the patients with MRD negativity at TP1 and TP2 and a 5-year RFS of 98\%; a high-risk group of $15 \%$ of patients with high MRD levels $\left(10^{-3}\right.$ or more) at both time points and a 5 -year RFS of $16 \%$; and an intermediate-risk group consisting of the $43 \%$ remaining patients with a 5-year RFS of $76 \%$ (updated RFS data). In this risk-group classification, TP1 appeared to be particularly useful for recognizing low-risk patients and TP2 for the recognition of high-risk patients, whereas later time points (particularly TP5) provided prognostic value for further classification of intermediate-risk patients. ${ }^{27}$ The above-described MRD-based risk group classification is used for treatment stratification in childhood ALL protocols of the BFM-Associazione Italiana di Ematologia ed Oncologia Pediatrica (AIEOP) (M.S., G.M., H.G., unpublished results, July 2000).

The aim of the present MRD study was to identify the different response groups within T-ALL and to elucidate the differences in overall treatment response between T-ALL and precursor B-ALL. From the cohort of patients included in the I-BFM-SG study, we were able to analyze 71 patients with T-ALL for their MRD pattern using one or 2 TCR gene rearrangements as PCR targets. These data were used to compare the presenting features and outcomes of the 71 patients with T-ALL with those of the previously reported 210 precursor B-ALL cases. ${ }^{27}$

A

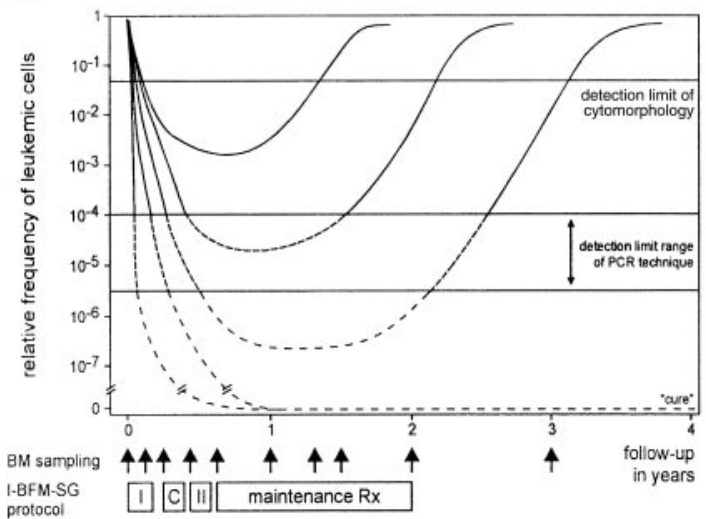

\section{Patients, materials, and methods}

\section{Patients and cell samples}

Bone marrow (BM) samples at diagnosis and as many as 9 time points during follow-up were collected from patients with childhood T-ALL included in the I-BFM-SG MRD study. Follow-up time points were: TP1, at the end of induction treatment (5 weeks); TP2, before consolidation (3 months); TP3, before reinduction treatment (6 months); TP4, before maintenance treatment (9 months); TP5, at 12 months; TP6, at 15 months; TP7, at 18 months; TP8, at the end of treatment (24 months); and TP9, at 1 year off treatment (36 months) (Figure 1A). ${ }^{27}$ Mononuclear cells (MNCs) were isolated from the collected $\mathrm{BM}$ samples and were stored in liquid nitrogen or at $-70^{\circ} \mathrm{C}$ for DNA extraction.

All children were treated according to protocols of the Austrian BFM Group (protocol ALL-BFM 90), the German BFM Group (protocol ALL-BFM 90), the AIEOP-ALL-91 protocol, or the Dutch Childhood Leukemia Study Group (DCLSG, protocol ALL-8). ${ }^{12,34,35}$ The 3 treatment protocols all had the same I-BFM-SG backbone. Patients with T-ALL were stratified into 2 treatment groups, medium-risk group (MRG) and high-risk group (HRG), according to the presenting features such as leukemic cell mass and prednisone response. ${ }^{12,34,35}$ All patient samples were obtained according to the informed consent guidelines of the local or national medical ethics committees.

The cohort of childhood ALL included in the I-BFM-SG MRD study from March 1991 to May $1995(\mathrm{n}=625)$ contained 74 patients with T-ALL (12\%); 30 had been previously analyzed. ${ }^{27}$ In the present study, 41 of the 44 remaining patients with T-ALL could be analyzed according to the same criteria-morphologic remission was reached at the end of induction treatment (at TP1), no BM samples were missing from more than 3 consecutive follow-up time points or from more than 4 follow-up time points in total, and at least one PCR target with a sensitivity of at least $10^{-4}$ was available for MRD detection. Of the total 71 patients with T-ALL, 24 had been treated in Germany, 20 in The Netherlands, 15 in Italy, and 12 in Austria.

The earlier analyzed group of precursor B-ALL was sufficiently large $(n=210)$ for the comparative T-ALL versus precursor B-ALL study and did not require the inclusion of extra patients. Five-year RFS rates of the analyzed T-ALL group $(\mathrm{n}=71)$ were $60 \%( \pm 6 \% \mathrm{SE})$ compared with $76 \%$ $( \pm 3 \%)$ in the precursor B-ALL group (Figure 1B). Survival of the T-ALL group studied is comparable to that of the total group of patients with T-ALL treated according to the same protocol. ${ }^{12,34,35}$ Patient characteristics of the 71 T-ALL and the 210 precursor B-ALL patients are given in Table 1.
B

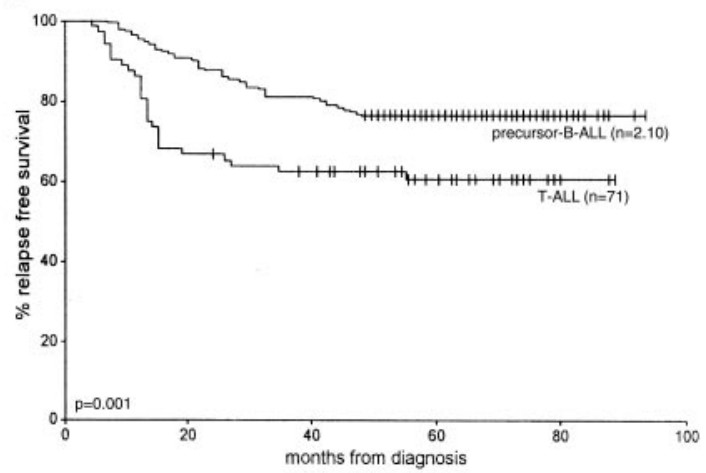

Figure 1. I-BFM-SG treatment protocol and RFS of T-ALL and precursor B-ALL. (A) Hypothetical graph shows the kinetics of leukemic cell decrease and regrowth in several ALL patients during and after treatment with the I-BFM-SG treatment protocol. MRD curves represent individual patients of the 3 MRD-based risk groups 27 -2 patients with slow MRD clearance and in the high-risk group; 2 patients with moderate MRD clearance and in the intermediate-risk group; and one patient with rapid MRD clearance in the low-risk group. The detection limit of cytomorphologic techniques and the detection-limit range of the PCR technique are indicated. Vertical arrows represent BM sampling time points at diagnosis, before each treatment block, at several time points during maintenance treatment, at cessation of therapy, and 1 year thereafter. I indicates induction treatment; C, consolidation treatment; II, reinduction treatment. (B) RFS of the 71 T-ALL and the 210 precursor B-ALL patients. Tick marks indicate censored survival times. 
Table 1. Patient group characteristics

\begin{tabular}{|c|c|c|}
\hline Parameters & $\begin{array}{c}\text { T-ALL } \\
(\mathrm{n}=71)\end{array}$ & $\begin{array}{l}\text { Precursor B-ALL } \\
\quad(n=210)\end{array}$ \\
\hline \multicolumn{3}{|l|}{ Treatment group distribution } \\
\hline SRG (\%) & 0 & $62(30)$ \\
\hline MRG (\%) & $50(70)$ & $135(64)$ \\
\hline HRG (\%) & $21(30)$ & $13(6)$ \\
\hline \multicolumn{3}{|l|}{ Sex } \\
\hline Male (\%) & $56(79)$ & $109(52)$ \\
\hline Female (\%) & $15(21)$ & $101(48)$ \\
\hline \multicolumn{3}{|l|}{ Age distribution in months } \\
\hline Mean (range) & $104(0-200)$ & $64(10-204)$ \\
\hline \multicolumn{3}{|l|}{ WBC count at diagnosis } \\
\hline Median $\times 10^{9} / \mathrm{L}$ (range) & $87(3-700)$ & $12(1-550)$ \\
\hline \multicolumn{3}{|l|}{ Prednisone response } \\
\hline Good (\%) & $54(76)$ & $200(95)$ \\
\hline Poor (\%) & $17(24)$ & $10(5)$ \\
\hline \multicolumn{3}{|c|}{ Five-year RFS rates $(\%)^{*}$ (no. relapses) } \\
\hline SRG & - & $84(10)$ \\
\hline MRG & $75(12)$ & $78(30)$ \\
\hline HRG & $24(16)$ & $23(10)$ \\
\hline All patients & $60(28)$ & $76(50)$ \\
\hline
\end{tabular}

Significant differences were found between T-ALL and precursor B-ALL for all parameters $(P \leq .001)$.

*Median relapse-free follow-up time of CCR patients is 63 months for T-ALL and 72 months for precursor B-ALL.

\section{Identification of PCR targets at diagnosis}

The junctional regions of TCR gamma (TCRG) and TCR delta (TCRD) gene rearrangements were used as patient-specific targets for PCR-based MRD detection. ${ }^{22,36,37}$ In addition, the so-called TAL1 deletions were used as PCR targets in some patients with T-ALL. ${ }^{22,38}$ Primers and PCR protocols were as described in the report of the BIOMED-1 Concerted Action BMH-CMT 94-1675.22 Clonal rearrangements in the TCRG, TCRD, and TAL1 genes were identified by heteroduplex PCR analysis and direct sequencing of the junctional regions with standardized sets of oligonucleotide primers. ${ }^{22,39}$ Based on the sequence data of the junctional regions, patient-specific oligonucleotides were designed for each identified MRDPCR target as described previously, using OLIGO software (W. Rychlik; Molecular Biology Insights, Cascade, CO). ${ }^{22}$

\section{PCR-based MRD detection during follow-up}

The MRD-PCR analyses of BM samples during follow-up of the patients with T-ALL were performed as described in the earlier BIOMED-1 report. ${ }^{22}$ Briefly, single PCR amplification with the standardized primer sets of the clone-specific rearrangement using $1 \mu \mathrm{g}$ DNA (equivalent to $10^{5}-10^{6}$ cells) was followed by dot blotting and hybridization with the corresponding ${ }^{32} \mathrm{P}$-labeled, patient-specific junctional region probe. Radioactive signals were evaluated using phosphor imaging. The sensitivity of each identified MRD-PCR target was established by use of a dilution experiment, in which DNA from the leukemic cells at diagnosis was diluted in 10-fold dilution steps into control DNA from a mixture of blood MNCs of 10 different healthy donors. Preferably, 2 independent PCR targets were used per patient, of which at least one reached a sensitivity of at least $10^{-4}$.

When MRD-PCR analysis of BM follow-up samples resulted in a hybridization signal, this time point was considered to be MRD positive. Consequently, if no signal was obtained, this time point was considered MRD negative, irrespective of the PCR target sensitivity. The frequency of leukemic cells in the BM samples during follow-up was estimated by comparison of the signals with those of the 10-fold dilution samples of DNA at diagnosis. This resulted in reproducible semiquantitative estimations of MRD-PCR results of $10^{-2}, 10^{-3}, 10^{-4}, 10^{-5}$, and $10^{-6}$ or more. If analysis of the same BM sample with 2 independent PCR targets resulted in different MRD level estimations, we assumed the highest MRD level to be the most accurate for data analysis, because lower MRD levels might be caused by subclone formation through continuing rearrangement processes. ${ }^{19,27}$ The following MRD levels were defined: high MRD levels of $10^{-2}$ or more, intermediate MRD levels of $10^{-3}$, and low MRD levels of $10^{-4}$ or less. MRD-PCR results of $10^{-5}$ and $10^{-6}$ were clustered with the $10^{-4}$ results because such low MRD levels were rarely found.

\section{Statistical analyses}

Relative frequencies were compared between groups using the $\chi^{2}$ of Fisher exact test. Between- and within-group comparisons of MRD levels were made using the Mann-Whitney $U$ and the Wilcoxon test, respectively. RFS according to the MRD results at the various time points was determined using Kaplan-Meier plots. Comparison of groups was performed using the $\log$ rank test or the log rank trend test for ordered groups (eg, MRD levels). Multivariate analysis of the predictive value of MRD at the various time points, allowing for different other variables (treatment group, age, sex, WBC on a continuous scale, and prednisone response) was performed using Cox regression analysis. It was further analyzed whether the prognostic effects of MRD levels differed between T-ALL and precursor B-ALL by investigating appropriate interaction terms in the regression models. $P \leq .05$ was considered significant.

\section{Results}

\section{Number of MRD-PCR targets}

In $66 \%$ (47 of 71 ) of the patients with T-ALL, 2 PCR targets (42 patients) or 3 PCR targets (5 patients) were used for MRD detection. The remaining 34\% of patients with T-ALL were analyzed by one MRD-PCR target. The 123 PCR targets used in the patients with T-ALL comprised 89 TCRG gene rearrangements, 10 incomplete TCRD gene rearrangements, 20 complete TCRD gene rearrangements, and 4 TAL1 deletions. In 76\% (93 of 123) of these targets, sensitivities of at least $10^{-4}$ were reached: 58 targets had a sensitivity of $10^{-4}, 28$ targets had $10^{-5}$, and 7 targets had $10^{-6}$.

In the precursor B-ALL group, 40\% (84 of 210) of patients were monitored with one MRD-PCR target and 60\% (126 of 210) were monitored with 2 targets (113 patients) or 3 targets (13 patients). Use of 1,2 , or 3 MRD-PCR targets did not significantly differ between T-ALL and precursor B-ALL $(P=.4)$.

\section{MRD in T-ALL is distinct from precursor B-ALL}

At the time of morphologic remission (TP1), that is, at 5 weeks of treatment, $80 \%$ (43 of 54) of patients with T-ALL were still MRD positive in contrast to $54 \%$ (78 of 144) patients with precursor

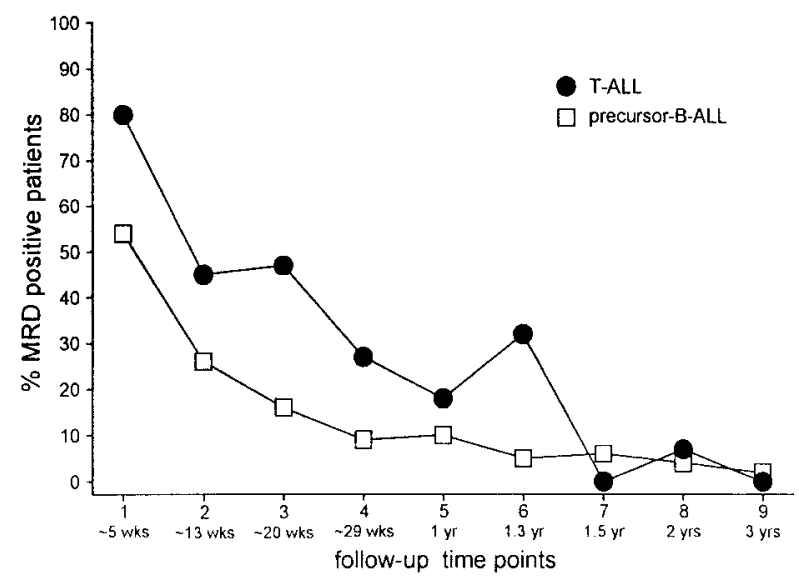

Figure 2. Frequencies. Frequency of MRD-positive patients at each individual follow-up time point for T-ALL and precursor B-ALL. 
B-ALL. In both groups the frequency of positive patients at TP2 (just before consolidation treatment) had approximately halved compared with the frequency found at TP1: $45 \%$ (25 of 55) in the T-ALL group and 26\% (40 of 156) in the precursor B-ALL group. A further decrease at later time points was similar in both groups, but the frequency of MRD-positive patients remained higher in patients with T-ALL compared with patients with precursor B-ALL until halfway through maintenance treatment (mean difference, $14 \% \pm 13 \%$ ) (Figure 2).

Not only the frequency of MRD-positive patients but also the level of MRD at the first 3 time points was significantly higher in T-ALL than in precursor B-ALL (all $P \leq .004$ ). In both groups, the MRD levels decreased from TP1 to TP2 $(P<.001)$. In precursor $\mathrm{B}-\mathrm{ALL}$, this reduction of MRD level was also observed from TP2 to TP3 $(P<.01)$. Such a decrease was not clear in patients with T-ALL $(P=.85)$, in whom most MRD levels were equal at TP2 and TP3, or when evaluated per patient (equal in $76 \%$ of patients).

MRD levels did not differ significantly between patients analyzed with one MRD-PCR target and patients with 2 or 3 MRD-PCR targets. This applied to all time points in T-ALL and precursor B-ALL.

\section{Prognostic value of MRD in T-ALL is higher than in precursor B-ALL}

At each follow-up time point, MRD levels were highly associated with subsequent relapse rates: the higher the MRD level, the worse the prognosis $(P[$ trend $]<.001)$. Patients with T-ALL $(\mathrm{n}=15)$ who were MRD negative or who had low MRD levels $\left(10^{-4}\right.$ or lower) at TP1 had a 5-year RFS rate of 100\%. The 5-year RFS rates of patients with MRD-negative precursor B-ALL at TP1 were $96 \%$ $( \pm 2 \%)$ and $74 \%( \pm 8 \%)$ for patients with low MRD levels (Figure $3)$. High MRD levels $\left(10^{-2}\right.$ or more) at TP1 resulted in a significantly lower 5-year RFS rates in T-ALL patients than in patients with precursor B-ALL-14\% $( \pm 8 \%)$ and $35 \%( \pm 11 \%)$, respectively $(P=.04)$. This difference in RFS between precursor B-ALL and T-ALL was also found at TP2 for high MRD levels $(P=.01)$ and intermediate MRD levels $\left(10^{-3}\right)(P=.05)$. At TP3, patients with MRD-negative T-ALL had a 5-year RFS rate of $97 \%$ $( \pm 3 \%)$ compared with $86 \%( \pm 3 \%)$ in precursor B-ALL (not significant), and patients with MRD-positive T-ALL had a 5-year RFS rate of $22 \%( \pm 8 \%)$ compared with $30 \%( \pm 8 \%)$ in precursor B-ALL (not significant). From TP2 onward, the discrimination between survival of MRD-positive and MRD-negative patients was always better in patients with T-ALL than in patients with precursor
A
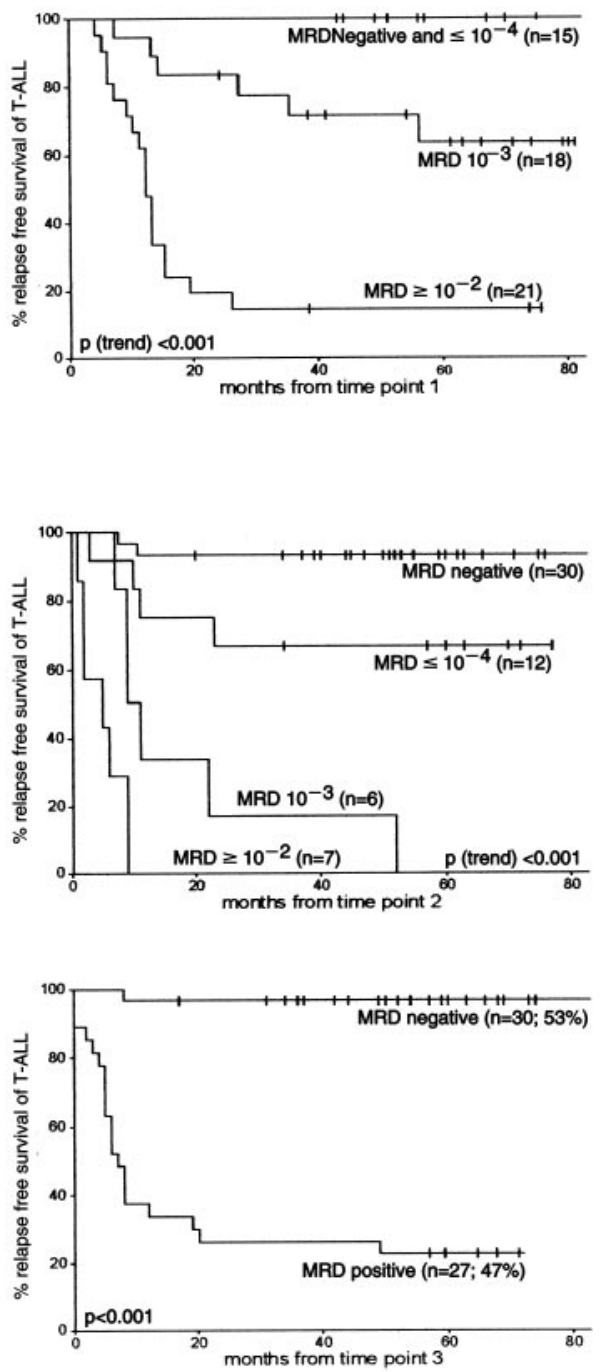

B
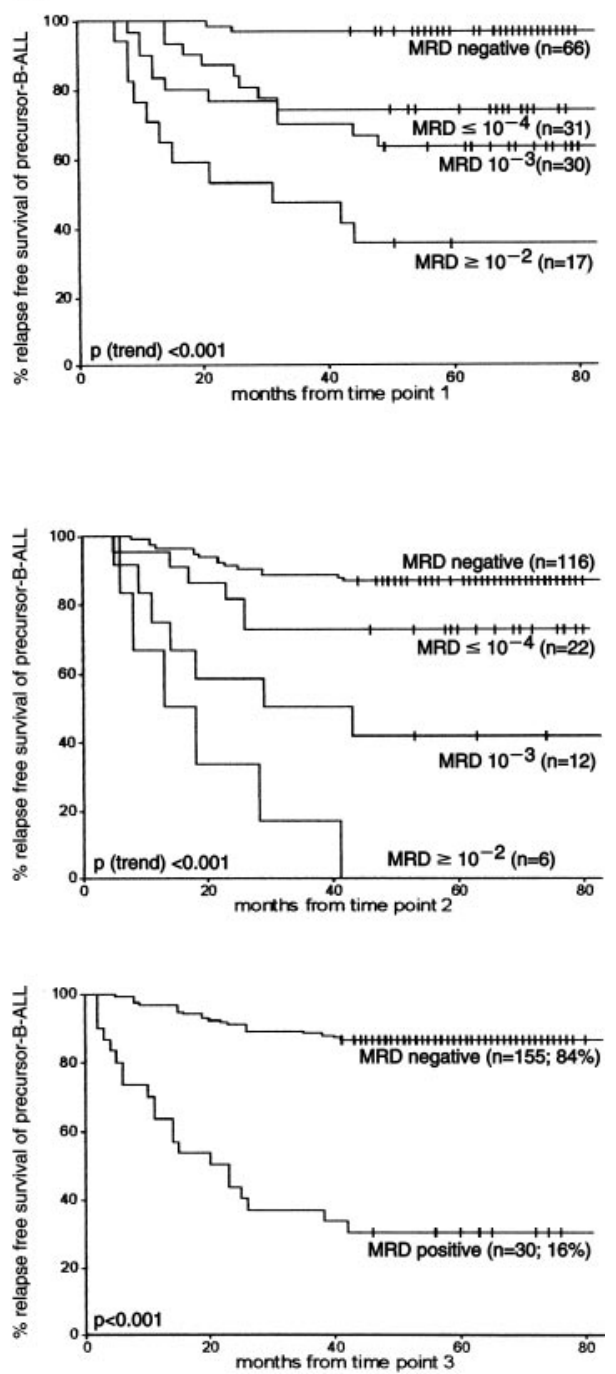

Figure 3. RFS according to MRD information at the first 3 follow-up time points in T-ALL and precursor B-ALL. (A) represents the MRD data obtained from T-ALL, and $(B)$ represents data from precurso B-ALL. The number of patients is given for each MRD level. Tick marks indicate censored survival times. 
B-ALL. Although apparent, these differences between T-ALL and precursor B-ALL were not always significant.

The prognostic value of MRD level differed significantly between T-ALL and precursor B-ALL at TP1 and TP2 $(P=.03$ and $P<.01$, respectively). Using Cox regression analysis, it was found that with each 10-fold decrease in MRD level at TP1 (from $10^{-2}$ or more to $10^{-3}$ to $10^{-4}$ or less to negative), the relapse rate was reduced by $84 \%$ in T-ALL compared with $62 \%$ in precursor B-ALL. This difference in gradient of RFS between T-ALL and precursor B-ALL was similar at TP2: for each drop in MRD level, the relapse rate was reduced by $79 \%$ in T-ALL compared with $61 \%$ in precursor B-ALL. At both time points, the observed differences in gradient of RFS between T-ALL and precursor B-ALL were not significantly affected by WBC count at diagnosis.

\section{MRD-based risk group classification}

The previously defined MRD-based risk group classification requires MRD information of TP1 and TP2. Patients at low risk were MRD negative at both time points, patients at high risk had MRD levels of $10^{-3}$ or higher at both time points, and the remaining patients formed the intermediate-risk group. ${ }^{27}$ Forty-three patients with T-ALL and 109 patients with precursor B-ALL could be analyzed at TP1 and TP2 and could consequently be classified. These patient subgroups did not differ significantly from the groups that lacked one or both time points with respect to distribution of age, sex, treatment group, and survival.

More than twice as many patients with T-ALL were classified in the MRD-based high-risk group than patients with precursor B-ALL-28\% (12 of 43) versus $11 \%$ (12 of 109), respectively $(P=.02)$. The size of the intermediate-risk group was comparable for T-ALL (49\%; 21 of 43) and precursor B-ALL (43\%; 47 of 109) patients. Consequently, fewer patients with T-ALL than patients with precursor B-ALL were classified at low risk: $23 \%$ of T-ALL (10 of 43) compared with $46 \%$ of precursor B-ALL (50 of 109) $(P=.01)$. In addition, the survival rates of the corresponding risk groups were similar: 5-year RFS rates in the low-risk group were $100 \%$ for T-ALL and $98 \%$ for precursor B-ALL $(P=.65)$. The 5 -year RFS in the intermediate-risk group was $76 \%$ for T-ALL and $77 \%$ for precursor B-ALL $(P=.78)$. A significant difference was found between the 5-year RFS rates for patients with T-ALL and patients with precursor B-ALL only in the high-risk group: $0 \%$ and $25 \%$, respectively $(P=.03)$ (Figure 4$)$.

The distribution of 1 versus 2 or 3 MRD-PCR targets did not significantly differ between the MRD-based risk groups of patients with T-ALL $(P=.28)$ and patients with precursor B-ALL $(P=.27)$, though the numbers of patients in some subgroups were low.

\section{Further classification of MRD-based intermediate-risk patients}

Later time points may have additional value for recognizing patients at good and poor risk in the MRD-based intermediate-risk group. MRD information of the BM sample taken just before reinduction treatment (TP3) did not have a significant additional value for patients with intermediate-risk precursor B-ALL $(P=.08)$. On the other hand, for patients with intermediate-risk T-ALL, at TP3 a significant difference in outcome was found between MRD-negative patients ( $\mathrm{n}=10,5$-year RFS of 100\%) and MRDpositive patients $(\mathrm{n}=8,5$-year RFS of $38 \%)(P=.004)$. TP4 (start of maintenance treatment) also seemed to be of additional value for the intermediate-risk T-ALL group in contrast to precursor B-ALL; MRD-negative patients with T-ALL $(\mathrm{n}=11)$ had a 5-year RFS of $82 \%$, and MRD-positive patients $(\mathrm{n}=3)$ had a 5-year RFS of $0 \%$ $(P=.002)$. As found previously, TP5 at 1 year is an interesting time point for further monitoring patients with ALL initially classified as at intermediate risk. This holds true for precursor B-ALL and for T-ALL. We found significant differences between MRD-positive and MRD-negative patients, despite low patient numbers $(P<.001$ for T-ALL and $P=.004$ for precursor B-ALL).

Because of the larger gradient of RFS according to MRD levels at TP1 and TP2 in T-ALL and the consistently lower relapse rates in the patients with MRD-negative T-ALL compared with patients with precursor B-ALL (Figure 3), one would expect it to be easier to discriminate between good and poor treatment responses in patients with T-ALL in the MRD-based intermediate-risk group. Indeed, the $10 \mathrm{~T}$-ALL patients with seemingly moderate treatment responses at TP1, as defined by low or intermediate MRD levels ( $\leq$ $10^{-3}$ ) but MRD negativity at TP2, had excellent outcomes ( 0 of 10 relapses). The corresponding group of patients with precursor B-ALL has a relatively high relapse rate (25\%; 7 of 28$)$.

\section{Association between MRD-based risk group classification and T-ALL maturational stage}

Based on immunophenotype, patients with T-ALL could be categorized as having immature $\mathrm{CD} 1^{-} / \mathrm{CD} 3^{-} \mathrm{T}-\mathrm{ALL}(\mathrm{n}=17), \mathrm{CD} 1^{+}$ T-ALL $(\mathrm{n}=37)$, and mature $\mathrm{CD} 1^{-} / \mathrm{CD} 3^{+}$T-ALL $(\mathrm{n}=17)$ with 5 -year RFS rates of $47 \%, 72 \%$, and $41 \%$, respectively. The 43 patients with T-ALL of the MRD-based risk group classification comprised 12 immature T-ALL, $25 \mathrm{CD}^{+}$T-ALL, and 6 mature
A

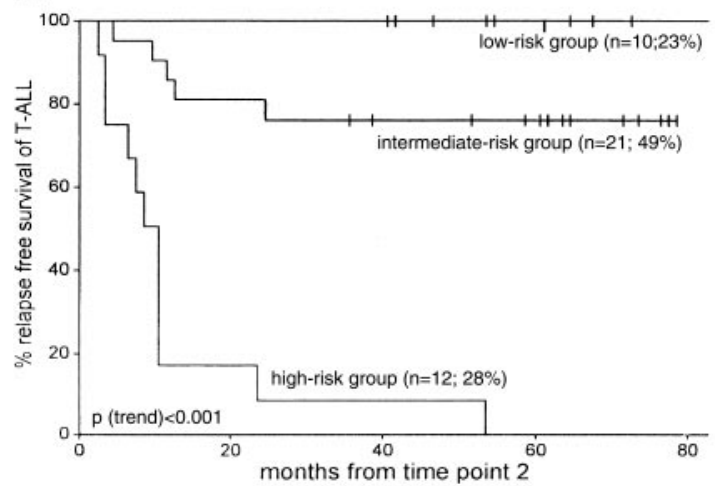

B

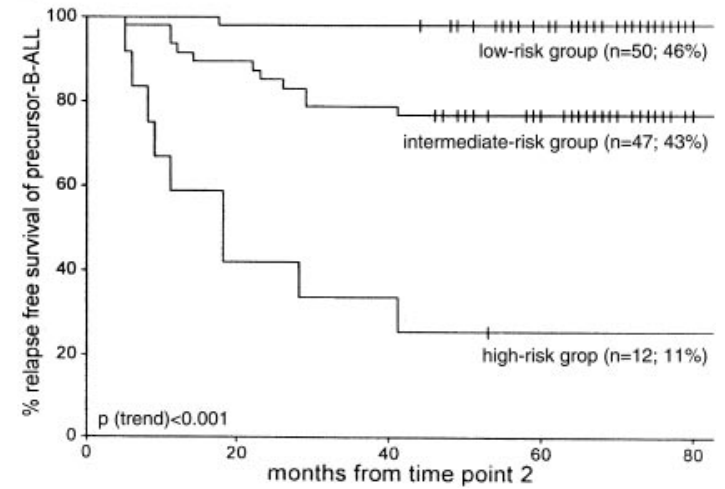

Figure 4. RFS according to the MRD-based risk group classification as defined by MRD information at TP1 and TP2. Patients in the low-risk group are characterized by MRD negativity at both time points, patients in the high-risk group are characterized by MRD levels $10^{-3}$ or greater at both time points, and the remaining patients form the intermediate-risk group. (A) 43 T-ALL patients. (B) 109 precursor B-ALL patients. For each MRD-based risk group, the number of patients and the relative size of each risk group are given. Tick marks indicate censored survival times. 
T-ALL. The $25 \mathrm{CD}^{+}{ }^{+} \mathrm{T}-\mathrm{ALL}$ patients were not randomly distributed over the MRD-based risk groups. In line with the relatively good prognosis of $\mathrm{CD}^{+} \mathrm{T}$-ALL, they represented $90 \%$ (9 of 10) of the MRD-based low-risk group and $57 \%$ (12 of 21) of the MRD-based intermediate risk group but only 33\% (4 of 12) of the MRD-based high-risk group $(P=.01)$. In addition, the 12 immature $\mathrm{CD} 1^{-} / \mathrm{CD} 3^{-}$T-ALL were not randomly distributed over the MRD-based risk groups: no immature patients with T-ALL were classified as low risk, whereas 5 were classified as MRD-based high risk and the remaining 7 were classified as intermediate risk. Despite the low patient numbers, these findings were in line with the difference in 5-year RFS between these 2 immunophenotypic subgroups. For the small group of mature $\mathrm{CD}^{+}$T-ALL patients $(n=6)$, no association with MRD-based risk groups was detected.

\section{Prognostic value of MRD in the T-ALL patient group}

Multivariate analysis was performed for the 71 patients with T-ALL. Simultaneous evaluation of MRD-based risk group and treatment group showed that both factors were significantly related to $\operatorname{RFS}(P<.001$ and $P=.03$, respectively). No additional prognostic value was found for age, sex, prednisone response, and WBC count on a continuous scale, although the latter weakly correlated with the MRD-based risk group classification.

\section{Discussion}

T-lineage ALL is associated with more high-risk features than precursor B-ALL. 2,4 Despite the improvement in outcome by intensive chemotherapy, patients with T-ALL generally have a significantly worse RFS than patients with precursor B-ALL. ${ }^{1,4,12,34}$ This difference in outcome was reflected by the MRD pattern when studying 9 follow-up time points taken during and after treatment by sensitive PCR techniques (detection limit $10^{-4}$ or less). At most time points the frequency of MRD-positive patients and the MRD levels were higher in the T-ALL group than in the precursor B-ALL group, reflecting the more frequent resistant disease in the T-ALL group.

Recently, MRD was proven to be the most important prognostic parameter in several large prospective studies. ${ }^{25-27}$ Now we confirm that also within the relatively small subgroup of T-ALL patients, MRD is a highly relevant prognostic factor. The impact of MRD level information differed significantly between T-ALL and precursor B-ALL at early time points, TP1 and TP2. For each 10-fold decrease in MRD level, approximately $80 \%$ fewer relapses were found in T-ALL compared with approximately $60 \%$ fewer relapses in precursor B-ALL.

Our results are consistent with those of previous MRD studies in T-ALL, which showed that MRD correlated with outcome. ${ }^{40,41}$ Dibenedetto et $\mathrm{al}^{41}$ found that the presence of MRD at the beginning of maintenance treatment was the strongest predictor of poor outcome. In our study MRD information at this relatively late time point was predictive. However, our semiquantitative MRD technique allowed us to discriminate between patients with good and poor prognoses at earlier time points, which is essential for early treatment stratification.

The currently applied MRD-based risk group classification in childhood ALL, which is based on the kinetics of tumor reduction during the first 3 months of treatment, was found to be valid for T-ALL as well. All patients with T-ALL who were classified in the MRD-based high-risk group subsequently relapsed. Half of the patients with T-ALL were classified as at intermediate risk with a 5 -year RFS rate of $76 \%$, and $23 \%$ of the patients with T-ALL were classified as at low risk with a $100 \%$ RFS rate. Survival in the intermediate-risk and low-risk groups was similar to that in precursor B-ALL; only survival in the high-risk group was different in T-ALL than in precursor B-ALL ( $0 \%$ vs $25 \%$ ). The distribution of T-ALL over the MRD-based risk groups was distinct from that of precursor B-ALL (Figure 4). More than twice as many patients with T-ALL were classified in the high-risk group (28\% vs $11 \%$ ), but the T-ALL low-risk group was half the size of the precursor B-ALL low-risk group ( $23 \%$ vs $46 \%$ ). Consequently, approximately half of the patients with T-ALL belonged in the intermediate-risk group. MRD monitoring at later time points (TP3, TP4, TP5) gave additional prognostic value for the patients with intermediate-risk T-ALL who were classified as good and poor risk based on the absence and presence of MRD at each time point, respectively. Further monitoring of intermediate-risk precursor B-ALL was less valuable (only TP5 gave a significant difference), probably reflecting the larger heterogeneity in this ALL subgroup.

Several BFM studies have shown the power of the initial prednisone response for recognition of many patients at high risk. ${ }^{8,9,12,29,42,43}$ However, our previous MRD study demonstrated that the prednisone response had no additional value on top of the MRD-based risk group classification. ${ }^{27}$ In addition, in this study, MRD information was a stronger prognostic factor than the prednisone response. Only 3 of 12 MRD-based high-risk precursor B-ALL patients and only 8 of 12 MRD-based high-risk T-ALL patients were predicted by poor prednisone response; all 11 patients had relapses. The other patients with poor prednisone response $(\mathrm{n}=8)$ all belonged to the MRD-based intermediate-risk group, and half of them ( 4 of 8 ) had relapses.

The distribution of patients with T-ALL over the MRD-based high-risk and low-risk groups seemed straightforward, showing either a resistant MRD pattern with $100 \%$ relapses or MRD negativity at TP1 and TP2 with $0 \%$ relapses, respectively. However, 10 intermediate-risk T-ALL patients with moderate MRD levels $\left(10^{-3}\right.$ or less) at TP1 and MRD negativity at TP2 also appeared to have excellent prognoses with no relapses. These observations are in contrast to patients with precursor B-ALL; $23 \%$ of MRD-based high-risk precursor B-ALL patients are still in continuous complete remission, and moderate MRD levels $\left(10^{-3}\right.$ or lower) at TP1 followed by MRD negativity at TP2 was still associated with $25 \%$ relapses in precursor B-ALL. This significant difference in prognostic value of MRD information between T-ALL and precursor B-ALL is in line with the larger gradient of RFS according to MRD levels at TP1 and TP2 in T-ALL (Figure 3) and the consistently lower relapse rates in the MRD-negative T-ALL patients at the first 5 follow-up time points.

When the 10 intermediate-risk T-ALL patients with moderate MRD levels at TP1 and MRD negativity at TP2 were shifted to the MRD-based low-risk group, the MRD-based risk group classification further improved-46\% (20 of 43) low-risk patients with $0 \%$ relapses, $26 \%$ (11 of 43 ) intermediate-risk patients with $45 \%$ relapses, and $28 \%$ (12 of 43) high-risk patients with $100 \%$ relapses. This remarkable separation of the 3 risk groups must be confirmed in larger series of patients with T-ALL. Nevertheless, we tried to understand the biologic significance of this observation and hypothesized that the MRD level at TP1 in patients with T-ALL might be influenced by the WBC count at diagnosis, because a good treatment response might not always result in MRD negativity at TP1 in patients with high initial WBC counts. We indeed found that the 10 T-ALL patients with MRD negativity at TP1 and TP2 had a low median WBC count of $25 \times 10^{9} / \mathrm{L}$, whereas the 10 patients with moderate MRD levels at TP1 $\left(10^{-3}\right.$ or less) but MRD negativity 
at TP2 had a higher median WBC count of $135 \times 10^{9} / \mathrm{L}$. This difference in WBC count was not statistically significant, possibly because of the relatively small number of patients.

Our MRD study shows that the MRD-based risk-group classification is highly valuable for T-ALL. Within 3 months of diagnosis, MRD analysis allowed the recognition of therapy-resistant T-ALL patients $(28 \%)$ with poor outcomes and a substantial group of T-ALL patients $(23 \%$ or even $47 \%$ ) with excellent prognoses. This implies that up to $75 \%$ of patients with T-ALL might be classified as either low-risk or high-risk patients. Although the overall survival rate of T-ALL is lower than that of precursor B-ALL, still $60 \%$ to $70 \%$ of patients with T-ALL are long-term survivors. ${ }^{15-17}$ Many of these survivors can now probably be recognized as low-risk T-ALL patients. These data further support the application of MRD kinetic information for treatment stratification in childhood ALL. It should be emphasized that for this purpose the MRD techniques should reach sensitivities of at least $10^{-4}$.

Independent from prognostic parameters at diagnosis, MRD analysis provides direct insight into the in vivo drug sensitivity of ALL cells. Our study shows that this type of information allows a highly accurate prediction of outcome within the 2 major immunophenotypic ALL categories, particularly in T-ALL. It can be anticipated that sensitive MRD analysis comprises a tool to define distinct risk groups within other seemingly homogeneous prognostic categories, such as leukemias with a particular chromosomal aberration.

\section{Acknowledgments}

We thank Annemarie Wijkhuijs and Daniëlle Jacobs (Rotterdam, Netherlands), Dorothee Erz, Yvonne Stark, and Simone Busenbender (Heidelberg, Germany), and Susanna Fischer and Marianne Konrad (Vienna, Austria) for their expert technical assistance. We thank the participants of the International BFM Study Group for their close collaboration in performing the MRD study, namely the Dutch Childhood Leukemia Study Group (P.J. van Dijken, K. Hählen, W.A. Kamps, E.F. van Leeuwen, F.A.E. Nabben, A. Postma, J.A. Rammeloo, G.A.M. de Vaan, A.J.P. Veerman, E.Th. van 't Veer-Korthof, and R.S. Weening), the German BFM Group (W. Dörffel, C. Niemeyer, F. Berthold, M. Rister, A. Jobke, M. Domula, H. Wehinger, K. Hofmann, F.J. Göbel, P. Heidemann, J.D. Beck, N. Graf, U. Mittler, A. Reiter, J.D. Thaben, G. Henze, R. Dickerhoff, J. Treuner, R. Geib, P. Exadactylos, U Bode, G. Eggers, W. Schröter, and C. Tautz), the Austrian BFM Group (B. Ausserer, F.M. Fink, R. Jones, G. Mann, G. Müller, I. Mutz, R. Ploier, W. Pobegen, K. Schmitt, and O. Stöllinger), and the Italian AIEOP Group (G. Masera, V. Conter, M.G. Valsecchi, M. Aricò, E. Madon, G. Basso, E. Barisone, L. Zanesco, M.C. Putti, M.T. Di Tullio, A. Murano, P.A. Macchia, and C. Favre). We thank Dr T. Szczepański, Dr K. Hählen, and Dr T. Révèsz for critical reading of the manuscript.

\section{References}

1. Ludwig WD, Reiter A, Löffler H, et al. Immunophenotypic features of childhood and adult acute lymphoblastic leukemia: experience of the German Multicentre Trials ALL-BFM and GMALL. Leuk Lymphoma. 1994;13:71-76.

2. Pui $\mathrm{CH}$, Behm FG, Singh B, et al. Heterogeneity of presenting features and their relation to treatment outcome in 120 children with T-cell acute lymphoblastic leukemia. Blood. 1990;75:174-179.

3. Uckun FM, Sensel MG, Sun L, et al. Biology and treatment of childhood T-lineage acute lymphoblastic leukemia. Blood. 1998;91:735-746.

4. Pui CH, Behm FG, Crist WM. Clinical and biologic relevance of immunologic marker studies in childhood acute lymphoblastic leukemia. Blood. 1993; 82:343-362.

5. Ludwig WD, Harbott J, Bartram CR, et al. Incidence and prognostic significance of immunophenotypic subgroups in childhood acute lympho blastic leukemia: experience of the BFM study 86. Recent Results Cancer Res. 1993;131:269282.

6. Niehues T, Kapaun P, Harms DO, et al. A classification based on $T$ cell selection-related phenotypes identifies a subgroup of childhood T-ALL with favorable outcome in the COALL studies. Leukemia. 1999;13:614-617.

7. Pullen J, Shuster JJ, Link M, et al. Significance of commonly used prognostic factors differs for children with $T$ cell acute lymphocytic leukemia (ALL), as compared to those with B-precursor ALL: a Pediatric Oncology Group (POG) study. Leukemia. 1999;13:1696-1707.

8. Riehm H, Reiter A, Schrappe M, et al. Corticosteroid-dependent reduction of leukocyte count in blood as a prognostic factor in acute lymphoblastic leukemia in childhood (therapy study ALL-BFM 83). Klin Pädiatr. 1987;199:151-160.

9. Reiter A, Schrappe M, Ludwig WD, et al. Chemotherapy in 998 unselected childhood acute lymphoblastic leukemia patients: results and conclusions of the multicenter trial ALL-BFM 86. Blood. 1994;84:3122-3133.

10. Steinherz PG, Gaynon PS, Breneman JC, et al. Cytoreduction and prognosis in acute lympho- blastic leukemia - the importance of early marrow response: report from the Childrens Cancer Group. J Clin Oncol. 1996;14:389-398.

11. Griffin TC, Shuster JJ, Buchanan GR, Murphy $\mathrm{SB}$, Camitta BM, Amylon MD. Slow disappearance of peripheral blood blasts is an adverse prognostic factor in childhood T cell acute lymphoblastic leukemia: a Pediatric Oncology Group study. Leukemia. 2000;14:792-795.

12. Schrappe M, Reiter A, Ludwig WD, et al. Improved outcome in childhood acute lymphoblastic leukemia despite reduced use of anthracyclines and cranial radiotherapy: results of trial ALL-BFM 90. Blood. 2000;95:3310-3322.

13. Arico M, Basso G, Mandelli F, et al. Good steroid response in vivo predicts a favorable outcome in children with T-cell acute lymphoblastic leukemia: the Associazione Italiana Ematologia Oncologia Pediatrica (AIEOP). Cancer. 1995;75:1684-1693.

14. Pieters R, Kaspers GJ, van Wering ER, et al. Cellular drug resistance profiles that might explain the prognostic value of immunophenotype and age in childhood acute lymphoblastic leukemia. Leukemia. 1993;7:392-397.

15. Consolino R, Legitimo A, Rondelli R, et al. Clinical relevance of CD10 expression in childhood ALL: the Italian Association for Pediatric Hematology and Oncology (AIEOP). Haematologica. 1998;83: 967-973.

16. Amylon MD, Shuster J, Pullen J, et al. Intensive high-dose asparaginase consolidation improves survival for pediatric patients with $\mathrm{T}$ cell acute lymphoblastic leukemia and advanced stage lymphoblastic lymphoma: a Pediatric Oncology Group study. Leukemia. 1999;13:335-342.

17. Reiter A, Schrappe M, Ludwig WD, et al. Intensive ALL-type therapy without local radiotherapy provides a $90 \%$ event-free survival for children with T-cell lymphoblastic lymphoma: a BFM group report. Blood. 2000;95:416-421.

18. Campana $\mathrm{D}, \mathrm{Pui} \mathrm{CH}$. Detection of minimal residual disease in acute leukemia: methodologic advances and clinical significance. Blood. 1995; 85:1416-1434

19. Szczepanski T, Orfão A, van der Velden VHJ, San
Miguel JF, van Dongen JJM. Minimal residual disease in leukaemia patients. Lancet Oncol. 2001; 2:409-417.

20. Szczepanski T, Langerak AW, Wolvers-Tettero ILM, et al. Immunoglobulin and T cell receptor gene rearrangement patterns in acute lymphoblastic leukemia are less mature in adults than in children: implications for selection of PCR targets for detection of minimal residual disease. Leukemia. 1998;12:1081-1088.

21. Szczepanski T, Beishuizen A, Pongers-Willemse $\mathrm{MJ}$, et al. Cross-lineage T-cell receptor gene rearrangements occur in more than ninety percent of childhood precursor-B-acute lymphoblastic leukemias: alternative PCR targets for detection of minimal residual disease. Leukemia. 1999;13: 196-205.

22. Pongers-Willemse MJ, Seriu T, Stolz F, et al. Primers and protocols for standardized MRD detection in ALL using immunoglobulin and T cell receptor gene rearrangements and TAL1 deletions as PCR targets: report of the BIOMED-1 Concerted Action: investigation of minimal residual disease in acute leukemia. Leukemia. 1999;13:110-118.

23. Jacquy C, Delepaut B, Van Daele S, et al. A prospective study of minimal residual disease in childhood B-lineage acute lymphoblastic leukaemia: MRD level at the end of induction is a strong predictive factor of relapse. Br J Haematol. 1997; 98:140-146.

24. Goulden NJ, Knechtli CJ, Garland RJ, et al. Minimal residual disease analysis for the prediction of relapse in children with standard-risk acute lymphoblastic leukaemia. Br J Haematol. 1998;100: 235-244.

25. Cave $\mathrm{H}$, van der Werff ten Bosch J, Suciu S, et al. Clinical significance of minimal residual disease in childhood acute lymphoblastic leukemia. N Engl J Med. 1998;339:591-598.

26. Coustan-Smith E, Behm FG, Sanchez J, et al. Immunological detection of minimal residual disease in children with acute lymphoblastic leukaemia. Lancet. 1998;351:550-554.

27. Van Dongen JJM, Seriu T, Panzer-Grumayer ER, 
et al. Prognostic value of minimal residual disease in acute lymphoblastic leukaemia in childhood. Lancet. 1998;352:1731-1738.

28. Ribeiro RC, Broniscer A, Rivera GK, et al. Phila delphia chromosome-positive acute lymphoblastic leukemia in children: durable responses to chemotherapy associated with low initial white blood cell counts. Leukemia. 1997;11:1493-1496.

29. Schrappe M, Arico M, Harbott J, et al. Philadelphia chromosome-positive $\left(\mathrm{Ph}^{+}\right)$childhood acute lymphoblastic leukemia: good initial steroid response allows early prediction of a favorable treatment outcome. Blood. 1998;92:2730-2741.

30. Zuna J, Hrusak O, Kalinova M, Muzikova K, Stary J, Trka J. TEL/AML1 positivity in childhood ALL: average or better prognosis? Czech Paediatric Haematology Working Group. Leukemia. 1999; 13:22-24.

31. Arico M, Valsecchi MG, Camitta B, et al. Outcome of treatment in children with Philadelphia chromosome-positive acute lymphoblastic leukemia. N Engl J Med. 2000;342:998-1006.

32. Cimino G, Elia L, Rapanotti MC, et al. A prospective study of residual-disease monitoring of the ALL1/AF4 transcript in patients with $\mathrm{t}(4 ; 11)$ acute lymphoblastic leukemia. Blood. 2000;95:96-101.

33. Fasching $\mathrm{K}$, Konig $\mathrm{M}$, Hettinger $\mathrm{K}$, et al. MRD lev- els during the first months of treatment indicate relapses in children with $\mathrm{t}(12 ; 21)$-positive ALL. Leukemia. 2000;14:1707-1708.

34. Conter V, Arico M, Valsecchi MG, et al. Intensive BFM chemotherapy for childhood ALL: interim analysis of the AIEOP-ALL 91 study: Associazione Italiana Ematologia Oncologia Pediatrica. Haematologica. 1998;83:791-799.

35. Kamps WA, Bökkerink JPM, Hakvoort-Cammel FGAJ, et al. Results of the DCLSG-study ALL8 (1991-1997): BFM-oriented treatment without cranial irradiation $(\mathrm{Cl})$ and comparing conventional oral and high doses intravenous 6-mercaptopurine [abstract]. Med Pediatr Oncol. 1999;33: 169.

36. Breit TM, Wolvers-Tettero ILM, Beishuizen A, Verhoeven M-AJ, van Wering ER, van Dongen JJM. Southern blot patterns, frequencies and junctional diversity of T-cell receptor $\gamma$ gene rearrangements in acute lymphoblastic leukemia. Blood. 1993;82:3063-3074.

37. Szczepanski T, Langerak AW, Willemse MJ Wolvers-Tettero ILM, van Wering ER, van Dongen JJM. T cell receptor gamma (TCRG) gene rearrangements in T cell acute lymphoblastic leukemia reflect "end-stage" recombinations: implications for minimal residual disease monitoring. Leukemia. 2000;14:1208-1214.
38. Breit TM Beishuizen A, Ludwig WD et al tal-1 deletions in T-cell acute lymphoblastic leukemia as PCR target for detection of minimal residual disease. Leukemia. 1993;7:2004-2011.

39. Langerak AW, Szczepanski T, van der Burg M, Wolvers-Tettero ILM, van Dongen JJM. Heteroduplex PCR analysis of rearranged T cell receptor genes for clonality assessment in suspect $\mathrm{T}$ cell proliferations. Leukemia. 1997;11:2192-2199.

40. Neale GA, Menarguez J, Kitchingman GR, et al. Detection of minimal residual disease in T-cell acute lymphoblastic leukemia using polymerase chain reaction predicts impending relapse. Blood. 1991;78:739-747.

41. Dibenedetto SP, Lo Nigro L, Mayer SP, Rovera G, Schiliro $G$. Detectable molecular residual disease at the beginning of maintenance therapy indicates poor outcome in children with T-cell acute lymphoblastic leukemia. Blood. 1997;90:12261232.

42. Riehm H, Gadner H, Henze G, et al. Results and significance of six randomized trials in four consecutive ALL-BFM studies. Hämatol Bluttransfus. 1990;33:439-450.

43. Dördelmann M, Reiter A, Borkhardt A, et al. Prednisone response is the strongest predictor of treatment outcome in infant acute lymphoblastic leukemia. Blood. 1999;94:1209-1217. 